\title{
Nonlinear diffusion for chemotaxis and birth-death process for Keller-Segel model
}

\author{
Mustafa Ali Dokuyucu ${ }^{1}$ and Ercan Celik ${ }^{2}$ \\ ${ }^{1}$ Department of Mathematics, Agri Ibrahim Cecen University, Agri, Turkey \\ ${ }^{2}$ Department of Mathematics, Ataturk University, Erzurum, Turkey
}

Received: 2 May 2016, Accepted: 30 May 2016

Published online: 23 June 2016.

\begin{abstract}
This paper seeks to establish the stability of the birth-death process in relation to the Keller-Segel Model. As well, it attempts to describe the stability of non-linear diffusion for chemotaxis. Attention will be on mass criticality results applying to the chemotaxis model. Afterwards, the analysis of the relative stability that stationary states exhibit is undertaken using the Keller-Segel system for the chemotaxis having linear diffusion. Standard linearization and separation of variables are the techniques employed in the analysis. The stability or instability of the analysed cases is demonstrated by the graphics. By using the critical results obtained for the models, the graphics are then compared with the rest.
\end{abstract}

Keywords: Chemotaxis, Keller-Segel model, birth-death process

\section{Introduction}

The motion of an entity is occurs randomly and focus of this paper is to discuss the motion. Normally, animals, chemicals, bacteria and cells move randomly. Past microscopic analysis exhibit various individuals as moving irregularly[4,6]. Diffusion is acknowledged as one of the various phenomena occurring naturally. Local chemical reactions arise due to reaction-diffusion systems. These reactions involve the transformation of objects into each other. As well, the systems influence diffusion that entails objects spreading out over a surface [1].

Numerous types of animals and types rely on their sense of smell to facilitate the conveyance of knowledge taking place within species. For instance, such arises in the female silk moth Bobmyx mori as well as in various species of deep-sea fish. The particular chemicals, which the species emit, constitute the pheromones. Such insights prompt the need to establish what chemotaxis entails. Hereby, chemotaxis involves the movements of organisms or cells because of the concentration fields of external chemicals $[2,3,5]$

$$
\left\{\begin{array}{l}
\rho_{t}=D \rho_{x x}-\chi\left(\rho a_{x}\right)_{x} \\
a_{t}=D_{a} a_{x x}+g(a, \rho) .
\end{array}\right.
$$

According to Keller-Segel, [8] the kinetics terms would be $g(a, n)=h \rho-k a$ where $h, k$ are positive constants. While $(h \rho)$ is rational to the number of amoebae $n,(-k a)$ introduces decay of attractant activity. One simple model is $f(n)=0$, which means that we ignored the amoebae production rate. The chemotactic term $\chi(a)$ can be taken as a constant $\chi$. Then the nonlinear system is written with the linear form to $g(a, n)$. The parameters $D, D_{a}, \chi$ are constants. $D$ and $D_{a}$ are the diffusion coefficient of the cells and $a$,respectively, $h$ and $k$ are positive constants. 


\section{Definition of problem}

In this chapter we will study chemotaxis using a non-linear diffusion model. If the system (1) is rearranged for non-linear diffusion, we get

$$
\left\{\begin{array}{l}
\frac{\partial \rho}{\partial t}=D \frac{\partial^{2} \rho^{\gamma}}{\partial x^{2}}-\chi \frac{\partial}{\partial x}\left(\rho \frac{\partial a}{\partial x}\right) \\
\frac{\partial a}{\partial t}=D_{a} \frac{\partial^{2} a}{\partial x^{2}}+h \rho-k a .
\end{array}\right.
$$

We consider $\gamma>1$. The parameters $D, D_{a}, \chi$ are constants. $D_{a}$ is the diffusion coefficient of a, h and $\mathrm{k}$ are positive constants.

\subsection{Initial and Boundary conditions}

The initial conditions for system (2) are

$$
\left\{\begin{array}{l}
\rho(x, 0)=\rho_{0}(x) \\
a(x, 0)=a_{0}(x),
\end{array}\right.
$$

The boundary conditions are,

$$
\left\{\begin{array}{l}
\rho_{x}(0, L)=0 \\
a_{x}(0, L)=0
\end{array}\right.
$$

Let $\left(\rho^{\infty}, a^{\infty}\right)$ be a constant steady state. Equation (2) then yields,

$$
h \rho^{\infty}-k a^{\infty}=0
$$

We get the steady state condition using the previous equation

$$
\left(\rho^{\infty}, a^{\infty}\right)=\left(\rho^{\infty}, \frac{h \rho^{\infty}}{k}\right)
$$

The conservation of total mass

$$
\int \rho(x, t) d x=\int \rho_{0}(x) d x
$$

and then the steady state $\rho^{\infty}$ will be determined by

$$
\int_{\Omega} \rho^{\infty} d x=M
$$

where $\Omega=L$. Then we can write,

$$
\left(\rho^{\infty}, a^{\infty}\right)=\left(\frac{M}{L}, \frac{h M}{k L}\right)
$$

\subsection{Linear analysis}

Now consider a perturbation of the linear system for $\rho(x, t)$ and $a(x, t)$

$$
\begin{aligned}
& \rho(x, t)=\rho^{\infty}+u(x, t) \\
& a(x, t)=a^{\infty}+v(x, t)
\end{aligned}
$$


When the system (2) is rearranged for $u$ and $v$,

$$
\left\{\begin{array}{l}
\frac{\partial u}{\partial t}=D \gamma\left(\rho^{\infty}\right)^{\gamma-1} \frac{\partial^{2} u}{\partial x^{2}}-\chi \rho^{\infty} \frac{\partial^{2} v}{\partial x^{2}} \\
\frac{\partial v}{\partial t}=D_{a} \frac{\partial^{2} v}{\partial x^{2}}+h u-k v
\end{array}\right.
$$

Using the technique of the separation variables, the system (5) re-written in matrix form as

$$
\partial_{t} U_{n}(t)=A_{n} U_{n}(t) \quad A_{n}:=\left(\begin{array}{cc}
-D \gamma\left(\rho^{\infty}\right)^{\gamma-1} \mu_{n}^{2} & \chi \rho^{\infty} \mu_{n}^{2} \\
h & -D_{a} \mu_{n}^{2}-k
\end{array}\right)
$$

where

$$
U_{n}(t)=\left(\begin{array}{l}
\alpha_{n}(t) \\
\beta_{n}(t)
\end{array}\right)
$$

We will now explore how to solve matrix form (6). This can be written,

$$
\left(\begin{array}{cc}
\sigma+D \gamma\left(\rho^{\infty}\right)^{\gamma-1} \mu_{n}^{2} & -\chi \rho^{\infty} \mu_{n}^{2} \\
-h & \sigma+D_{a} \mu_{n}^{2}+k
\end{array}\right)\left(\begin{array}{c}
\alpha_{0} \\
\beta_{0}
\end{array}\right) e^{\sigma t}=0
$$

If the matrix in equation (7) is zero it can be written

$$
\left(\sigma+D \gamma\left(\rho^{\infty}\right)^{\gamma-1} \mu^{2}\right)\left(\sigma+D_{a} \mu^{2}+k\right)-h \chi \rho^{\infty} \mu^{2}=0
$$

where $\mu_{n}=\mu$. Let us re-write (8) as a quadratic in $\sigma$ :

$$
\sigma^{2}-\left(t r A_{n}\right) \sigma+\operatorname{det}\left(A_{n}\right)=0
$$

where

$$
\left\{\begin{array}{l}
\operatorname{Tr}\left(A_{n}\right)=-D \gamma\left(\rho^{\infty}\right)^{\gamma-1} \mu^{2}-D_{a} \mu^{2}-k<0 \quad \forall \mu \in \Re \\
\operatorname{det}\left(A_{n}\right)=\mu^{2}\left(D D_{a} \gamma\left(\rho^{\infty}\right)^{\gamma-1} \mu^{2}+D \gamma\left(\rho^{\infty}\right)^{\gamma-1} k-\chi \rho^{\infty} h\right) .
\end{array}\right.
$$

Thus there are two possibilities which are,

(i) $\sigma_{1}$ and $\sigma_{2}$ are negative,

(ii) $\sigma_{1}$ is negative and $\sigma_{2}$ is positive.

Since $\operatorname{det}\left(A_{n}\right)>0$ for all $\mu \in R$, and therefore the eigenvalues of $A_{n}$ are both strictly negative for all $\mu$. Therefore,

$$
D \gamma\left(\rho^{\infty}\right)^{\gamma-1} k-\chi \rho^{\infty} h>0
$$

where $\rho^{\infty}=\frac{M}{L}$. Then we get,

$$
\frac{D}{\chi}>\frac{h}{k}\left(\frac{M}{L}\right)^{(-\gamma+2)}
$$

On the other hand, if

$$
\frac{D}{\chi}<\frac{h}{k}\left(\frac{M}{L}\right)^{(-\gamma+2)}
$$

It is known that $\operatorname{det}\left(A_{n}\right)<0$. Therefore, $A_{n}$ has one positive eigen value, which implies linear instability. If the ratio $D / \chi$ is large enough then diffusion dominates and the sytem is stable, whereas if $D / \chi$ is small enough then chemotaxis dominates and the system is unstable. 


\subsection{Results}

Using the solution of equation (9), real part of growth rate $\left(\sigma_{1,2}\right)$, versus the wavenumber are plotted in Figure (1),(2) and (3). In Figure (1) and (2) are strictly stable for both lines. The critical result is that the red line decreases while the value of $\gamma$ rises.

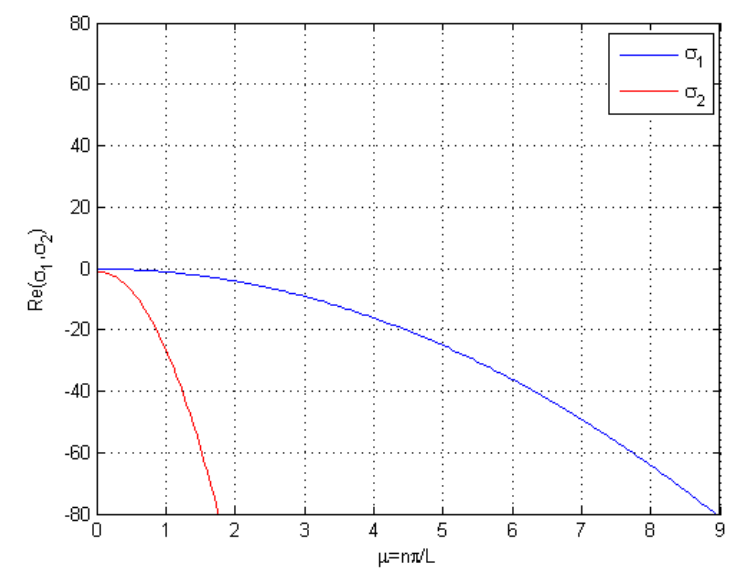

Fig. 1: $D=6.4, h=2, \chi=5, M / L=2, D_{a}=1, k=1$, and $\gamma=2$.

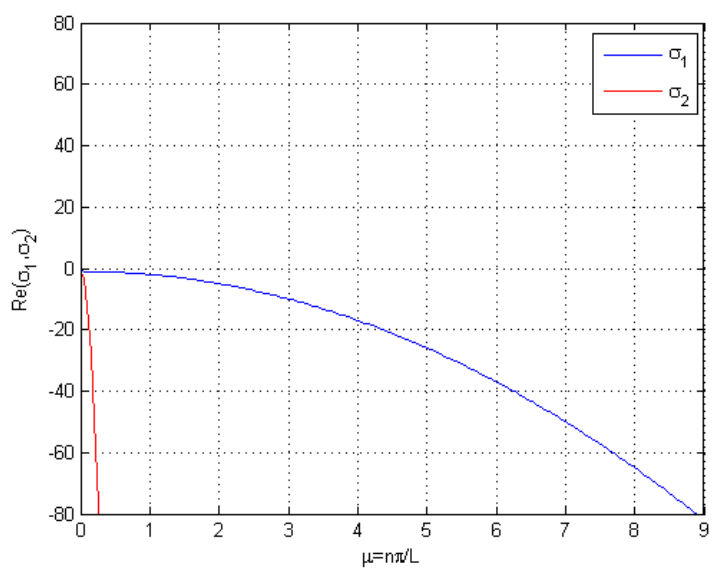

Fig. 2: $D=6.4, h=2, \chi=5, M / L=2, D_{a}=1, k=1$, and $\gamma=4$.

On the other hand, the blue line is unstable but after a some point this line became again stable. If,

$$
\frac{D}{\chi}<\frac{h}{k}\left(\frac{M}{L}\right)^{2}
$$




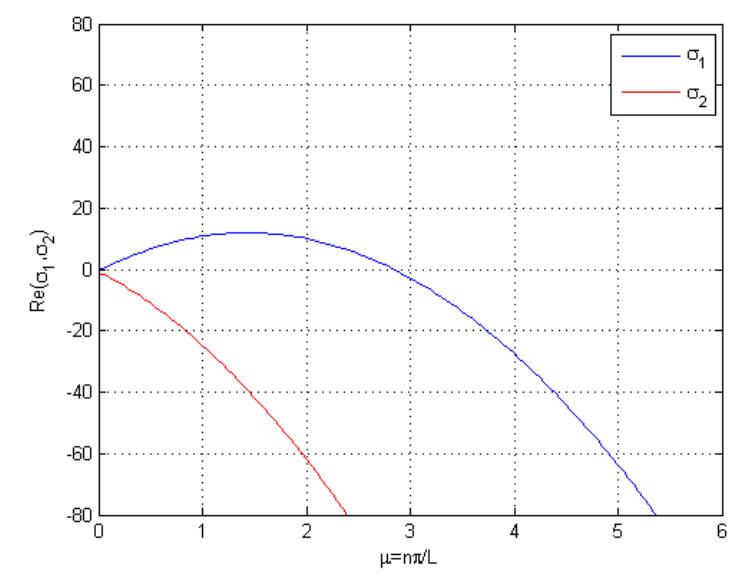

Fig. 3: $D=1.1, h=22, \chi=12, M / L=1.2, D_{a}=5, k=1.4$, and $\gamma=4$.

\section{Birth-death process}

In the previous chapter we ignored the birth-death process. Now, we will define a new system that includes birth-death term. The similar model was studied by Keller-Segel. They [7] introduced a simple model for slime mould aggregation and this model was developed by Myerscough, Maini, Murray and Winters.[9] We will introduce our model:

$$
\left\{\begin{array}{l}
\rho_{t}=D\left(\rho^{\gamma}\right)_{x x}-\chi \rho a_{x x}+r \rho\left(1-\frac{\rho}{\rho^{\infty}}\right) \\
a_{t}=D_{a} a_{x x}+h \rho-k a
\end{array}\right.
$$

where $D, D_{a}, \rho^{\infty}, \chi, h, k$ are positive constants. The initial conditions are the same in the previous chapter. The Neumann boundary conditions are used, namely $s(x) . \nabla \rho(x, t)=0$ and $s(x) \nabla a(x, t)=0$ where $s(x)$ is the outward unit normal to the boundary of the domain, $\partial \Omega$.

\subsection{Linear analysis and results}

Let us consider a small perturbation of the system (13) for $\rho$ anf $a$.

$$
\begin{aligned}
& \rho(x, t)=\rho^{\infty}+u(x, t) \\
& a(x, t)=a^{\infty}+v(x, t)
\end{aligned}
$$

Rearranging system (13):

$$
\left\{\begin{array}{l}
u_{t}=D \gamma\left(\rho^{\infty}\right)^{\gamma-1} u_{x x}-\chi \rho^{\infty} v_{x x}-r u \\
v_{t}=D_{a} v_{x x}+h u-k v .
\end{array}\right.
$$

Let us solve the system (14) in the form:

$$
\left(\begin{array}{l}
u \\
v
\end{array}\right)=\left(\begin{array}{c}
\alpha_{0} \\
\beta_{0}
\end{array}\right) e^{i \mu x+\sigma t}
$$


Re-writing system (14) as a matrix system, we get:

$$
\sigma\left(\begin{array}{l}
\alpha_{0} \\
\beta_{0}
\end{array}\right) e^{i \mu x+\sigma t}=\left(\begin{array}{cc}
-D \gamma\left(\rho^{\infty}\right)^{\gamma-1} \mu_{n}^{2}-r & \chi \rho^{\infty} \mu_{n}^{2} \\
h & -D_{a} \mu_{n}^{2}-k
\end{array}\right)\left(\begin{array}{l}
\alpha_{0} \\
\beta_{0}
\end{array}\right) e^{i \mu x+\sigma t}
$$

or equivalently

$$
\left(\begin{array}{cc}
\sigma+D \gamma\left(\rho^{\infty}\right)^{\gamma-1} \mu_{n}^{2}+r & -\chi \rho^{\infty} \mu_{n}^{2} \\
-h & \sigma+D_{a} \mu_{n}^{2}+k
\end{array}\right)\left(\begin{array}{l}
\alpha_{0} \\
\beta_{0}
\end{array}\right) e^{\sigma t}=0
$$

Solutions of the linearised system exist if the determined of this matrix is zero, that is

$$
\left(\sigma+D \gamma\left(\rho^{\infty}\right)^{\gamma-1} \mu^{2}+r\right)\left(\sigma+D_{a} \mu^{2}+k\right)-h \chi \rho^{\infty} \mu^{2}=0 .
$$

Let us re-write (17) as a quadratic in $\sigma$ :

$$
\sigma^{2}-\left(t r A_{n}\right) \sigma+\operatorname{det}\left(A_{n}\right)=0
$$

where

$$
\left\{\begin{array}{l}
\operatorname{Tr}\left(A_{n}\right)=-D \gamma\left(\rho^{\infty}\right)^{\gamma-1} \mu^{2}-D_{a} \mu^{2}-k-r \\
\operatorname{det}\left(A_{n}\right)=\mu^{2}\left(D D_{a} \gamma\left(\rho^{\infty}\right)^{\gamma-1} \mu^{2}+D \gamma\left(\rho^{\infty}\right)^{\gamma-1} k+D_{a} r-h \chi \rho^{\infty}\right)+r k
\end{array}\right.
$$

We now need to consider the next solution of (18). If both eigenvalues are negative, $\operatorname{det}\left(A_{n}\right)$ is greater than zero and it can be written as

$$
\operatorname{det}\left(A_{n}\right)=D D_{a} \gamma\left(\rho^{\infty}\right)^{\gamma-1} \mu^{4}+\left(D \gamma\left(\rho^{\infty}\right)^{\gamma-1} k+D_{a} r-h \chi \rho^{\infty}\right) \mu^{2}+r k>0 .
$$

When $\operatorname{det}\left(A_{2}\right)>0$ the red line is always stable, the blue line is unstable but the blue line also stable for very small $\mu$ and for large $\mu$. On the other hand, if we choose suitable parameters, the system can be made stable. In order to find these

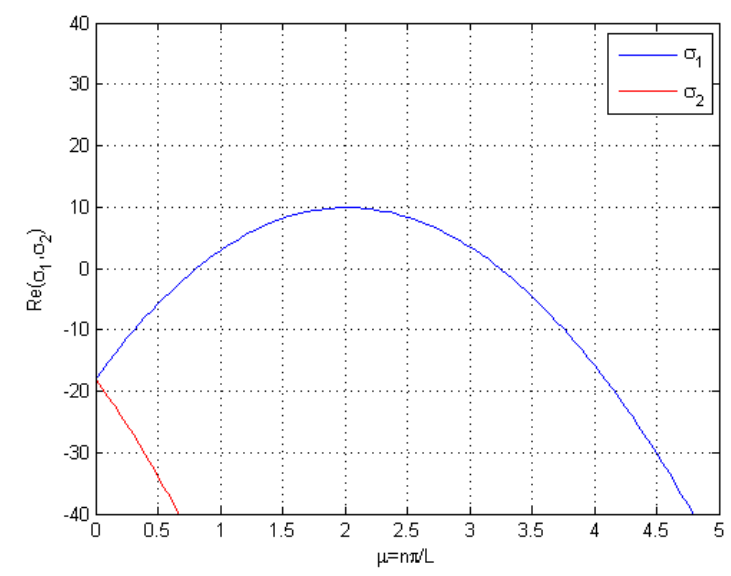

Fig. 4: $D=1.5, D_{a}=13, h=7.37, \chi=12, M / L=6.35, k=14, r=14$ and $\gamma=2$

requirements, we need to solve equation (20). If $\operatorname{det}\left(A_{n}\right)=0$, we get

$$
D D_{a} \gamma\left(\rho^{\infty}\right)^{\gamma-1} \mu^{4}+\left(D \gamma\left(\rho^{\infty}\right)^{\gamma-1} k+D_{a} r-h \chi \rho^{\infty}\right) \mu^{2}+r k=0 .
$$


Let us first consider a single, repeated root of the dispersion curve. The discriminat for this equation,

$$
D \gamma\left(\rho^{\infty}\right)^{\gamma-1} k+D_{a} r-h \chi \rho^{\infty}=-2 \sqrt{D D_{a} \gamma\left(\rho^{\infty}\right)^{\gamma-1} r k}
$$

Substituting equation (22) into equation (21) we get

$$
\mu^{4}-2 \sqrt{\frac{r k}{D D_{a} \gamma\left(\rho^{\infty}\right)^{\gamma-1}}}+\frac{r k}{2 D D_{a} \gamma\left(\rho^{\infty}\right)^{\gamma-1}}=0
$$

or equaivanetly

$$
\left(\mu^{2}-\sqrt{\frac{r k}{D D_{a} \gamma\left(\rho^{\infty}\right)^{\gamma-1}}}\right)^{2}=0 \Rightarrow \frac{n^{2} \pi^{2}}{L^{2}}=\sqrt{\frac{r k}{D D_{a} \gamma\left(\rho^{\infty}\right)^{\gamma-1}}}
$$

where $\mu=n \pi / L$.

It is clearly seen that both lines are stable in Figure (5).

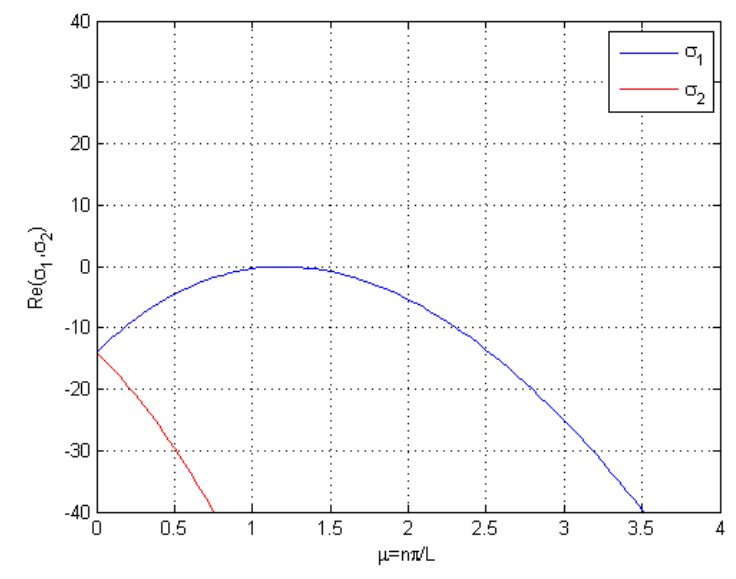

Fig. 5: $D=1.5, D_{a}=13, h=21, \chi=12, M / L=2.44, k=14, r=14$, and $\gamma=2$.

\section{Conclusion}

In this paper, we analysed chemotaxis via Keller-Segel model. During the anaylsis, we had some main conclusion. We studied non-linear diffusion for chemotaxis with the Neumann boundary conditions. We can say that stability and instability depend on the ratio $D / \chi$. It means that while the values $\chi$ and $h$ must be lagre, the values $D$ and $k$ must be small for the non-linear model. We also analysed the birth-death process. It can be seen that, when the value $h$ is enough large and the value $\chi$ is enough small, the system is always stable.

\section{References}

[1] J.D. Murray, Mathematical Biology I: an Introduction, 3rd. edn., Interdisciplinary Applied Mathematics,17 405-406,(2002)

[2] T. Hofer, Chemotaxis and aggregation in the cellular slime mould, Berlin, 137-150,(1999) 
[3] D. Horstman, From 1970 until present: the Keller-Segel model in chemotaxis and its consequences, I. Jahresberichte DMV. 105(3), 103-165,(2003)

[4] D. Horstman, Lyapunov functions and L p-estimates for a class of reaction diffusion systems, Coll. Math. 87,113-127,(2001)

[5] B. Perthame, Transport Equations in Biology, Birkhauser, (2007).

[6] T. Hillen and K.J. Painter, A user's guide to PDE models for chemotaxis. Journal of Mathematical Biology, 58,183-217,(2009)

[7] E.F. Keller and L.A. Segel, Initiation of slime mold aggregation viewed as an instability, J. Theor. Biol. 26,399-415,(1970)

[8] E.F. Keller and L.A. Segel, Model for chemotaxis, J. Theor. Biol. 30,225-234,(1971).

[9] M. R. Myerscough, P. K. Maini, J.D. Murray and K. H. Winters, Two dimensional pattern formation in a Chemotaxis system, In dynamics of complex interconnected biological system, 65-83, (1990) 